\title{
LEBENSLANGES LERNEN ALS FOLGE SOZIO-ÖKONOMISCHER TRENDS
}

\author{
Michael Klotz
}

FH Stralsund

Received: 10 February, 2003

\begin{abstract}
Modern societies are faced with lifelong learning as an important challenge. Lifelong learning must be structured and its content must be defined. In order to do this the factors, which influence the need for lifelong learning must be known. These factors - examples are globalization, new markets, new competition, the information society, and knowledge management - will be presented in this article.
\end{abstract}

Keywords: learning, knowledge management, globalization

\section{Einführung}

Der Begriff des lebenslangen Lernens basiert auf der generellen Entwicklung, dass in modernen Gesellschaften die Komplexität von Arbeits- und Lebenswelten in den beiden zurück liegenden Jahrzehnten in hohem Maße gestiegen ist. Schlagworte wie Fragmentierung, Globalisierung und Informatisierung stehen für diesen Sachverhalt.

Der Einzelne - aber auch Gruppen und Gesellschaften insgesamt - können dieser gestiegenen Komplexität mit Wissen, das im Rahmen der Schule und der Berufsausbildung einmalig erlangt wurde, nicht mehr gerecht werden. Stattdessen werden die kontinuierliche Anpassung der eigenen Fähigkeiten und Fertigkeiten an den aktuellen Wissensstand sowie das Erlernen neuer Qualifikationen erforderlich. So wird z. B. auch im traditionellen Ingenieursberuf die Ergänzung um soziale Schlüsselqualifikationen immer dringlicher, und der Tätigkeitsbereich erweitert sich um Umweltthemen, aber auch um soziale, gesellschaftliche Belange. Weiterhin werden Managementqualitäten bis hin zum unternehmerischen Denken und Handeln gefordert (nach [1]).

Die Notwendigkeit eines lebenslangen Lernens wird heute nicht mehr bestritten, sondern von vielen gesell- schaftlichen Gruppierungen betont oder gar gefordert. So betont beispielsweise die Europäische Kommission, dass das Lernen vom Kindergarten bis ins Rentenalter Lernumwelten erfordert, in denen sich alle lernwilligen Menschen diejenigen Kenntnisse und Fertigkeiten aneignen können, die ihren Bedürfnissen entsprechen (vgl. [2]).

Die Herausforderung des lebenslangen Lernens kann jedoch nur dann gemeistert werden, wenn die zugrunde liegenden sozio-ökonomischen Trends als Bestimmungsfaktoren bekannt sind und verstanden werden. Denn dies ist die Voraussetzung dafür, um ein lebenslanges Lernen situationsspezifisch und problemadäquat in seinen Strukturen und Inhalten gestalten zu können. Dieser Beitrag soll primär aus Unternehmenssicht die wesentlichen Faktoren beleuchten, aus denen die Erfordernis eines lebenslangen Lernens resultiert.

\section{Bestimmungsfaktoren des lebenslangen Lernens}

Die Notwendigkeit des lebenslangen Lernens ist nicht auf einen einzigen oder wenige Gründe zurück zu führen. Viele Entwicklungen beeinflussen diesen generellen Trend. Abbildung 1 zeigt die wesentlichen 


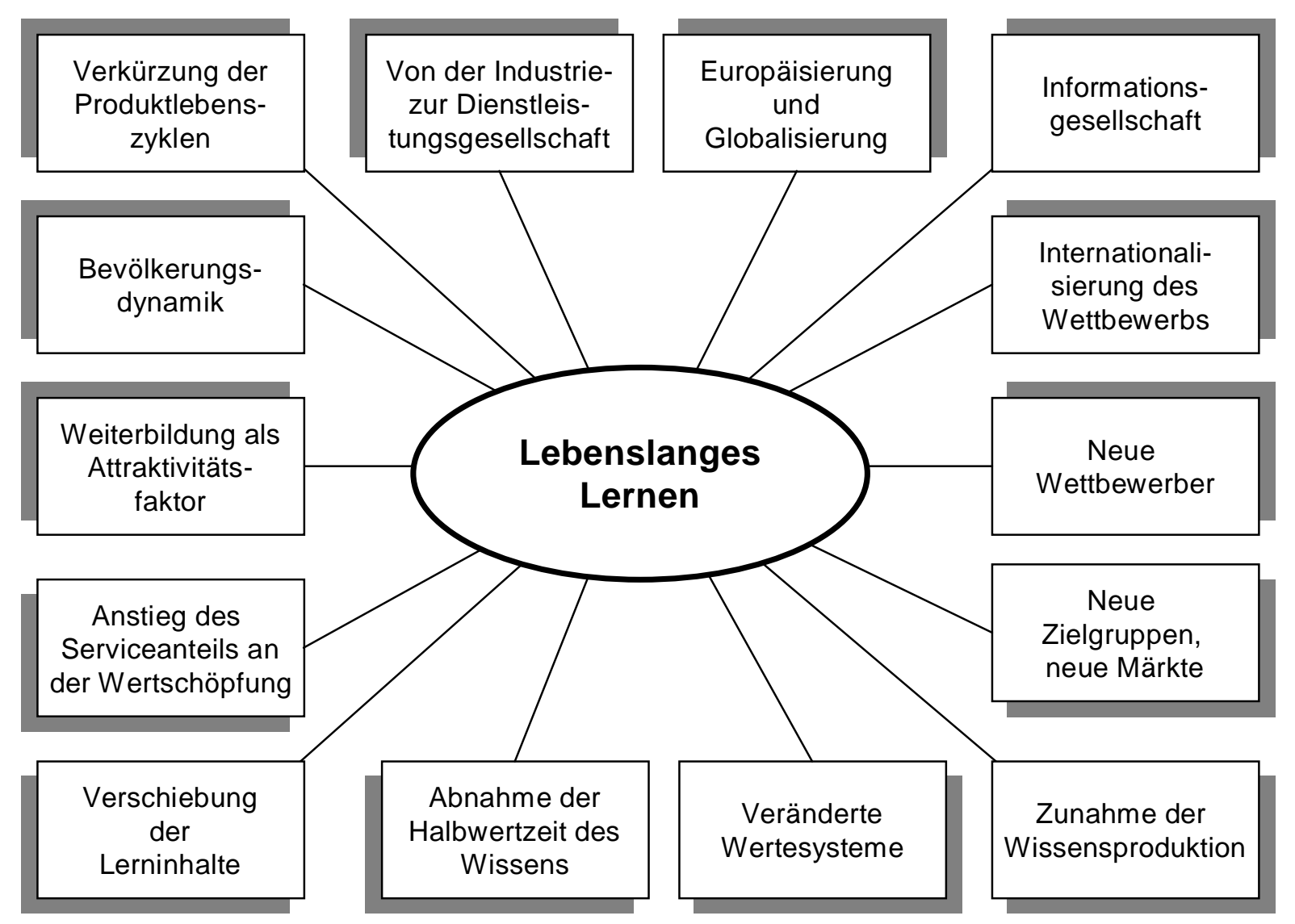

Abb. 1: Bestimmungsfaktoren des lebenslangen Lernens (nach [3])

Bestimmungsfaktoren, die ein lebenslanges Lernen erforderlich machen.

\subsection{Europäisierung und Globalisierung}

In der Arbeitswelt zeigt sich die Globalisierung vor allem in den internationalen Waren-, Informations- und Geldströmen. So ist der Weltaußenhandel (nominell) von 57,5 Mrd. US-\$ im Jahr 1948 auf 5.334,6 Mrd. US-\$ im Jahr 1998 gestiegen [4]. Die finanzielle Globalisierung zeigt sich bspw. im Wachstum der Tagesumsätze auf den internationalen Devisenmärkten. Diese sind von 79 Mio. US-\$ im Jahr 1979 über 500 Mio. US-\$ im Jahr 1990 auf heute nunmehr 1,5 Billionen US-\$ gestiegen (s. [5]). Diese Austauschbeziehungen basieren auf Arbeitsleistungen, die gemeinsam von Mitarbeitern unterschiedlicher Nationalitäten, Mentalitäten und Sprachräume erbracht werden.

Mit der Internationalisierung im Rahmen eines geeinten, erweiterten Europas und der Globalisierung sind kollektive Identitätskonstruktionen in Bewegung gekommen, so dass Bildungsprozesse verschiedenster Art notwendig werden. Sprachkompetenz und die
Kenntnis der Sitten und Gebräuche anderer Kulturen sind hierbei nur die Spitze des Eisberges. Vor allem zur Schaffung der mentalen und intellektuellen Voraussetzungen für eine interkulturelle Zusammenarbeit sind künftig immense Bildungsanstrengungen notwendig.

\subsection{Informationsgesellschaft}

Der Wandel zur Informationsgesellschaft stellt nicht nur für IT-, Medien- und Kommunikationsunternehmen, sondern für alle Wirtschaftsbereiche eine große Herausforderung dar. Der bundesdeutsche Markt für Informations- und Kommunikations- (IuK) technik ist 2001 auf über 137 Mrd. $€$ gewachsen (nach [6]) und bildet damit einen der größten Wirtschaftszweige Deutschlands. Dies ist die Folge einer zunehmend vollständigen Unterstützung aller Geschäftsprozesse in Unternehmen durch integrierte Informations- und Kommunikationstechnologien. Da letztlich alle Mitarbeiter die IuK- Technik als Anwender nutzen müssen, wird eine entsprechende Medienkompetenz der Mitarbeiter zum Erfolgsfaktor im Wettbewerb. Es ist gerade die in Abbildung 2 dargestellte Technologiekonver- 
genz, aus der ein kontinuierlicher Bedarf zur Aktualisierung der Medienkompetenz resultiert.

Aber nicht nur die Erhöhung der Effizienz steht auf der Tagesordnung. Unternehmen müssen auf die sich ändernden Bedürfnisse der Menschen reagieren und Information als Wirtschaftsgut derart nutzen, dass zukunftsfähige Informations- und Medienprodukte entwickelt werden. Dies erfordert kompetente und kreative Produktentwickler und -designer, die über den neuesten IuK- Wissensstand verfügen.

\subsection{Internationalisierung des Wettbewerbs}

Internationalisierung und ein sich verschärfender Wettbewerb im internationalen Maßstab sind grundlegende Entwicklungen unserer Zeit. Verstärkt wird dieser Trend durch das politische Zusammenwachsen Europas. Aber auch in Übersee, wie beispielsweise in Indien oder China, öffnen sich gewaltige Märkte und regen den Welthandel an.

So ist das chinesische Bruttoinlandsprodukt 2001 um $7,3 \%$ gewachsen, wobei sich die Importe auf 232 Mrd. US-\$ beliefen, für 2003 werden $320 \mathrm{Mrd}$. US-\$ prognostiziert (nach [7]). Gerade das in ostasiatischen Staaten nach wie vor gute Image deutscher Produkte bietet auch mittelständischen Unternehmen große Chancen, neue Märkte zu erobern - wenn die Mitarbeiter entsprechend qualifiziert sind.

\subsection{Neue Wettbewerber}

Neue Wettbewerber auch und gerade aus Wirtschaf-

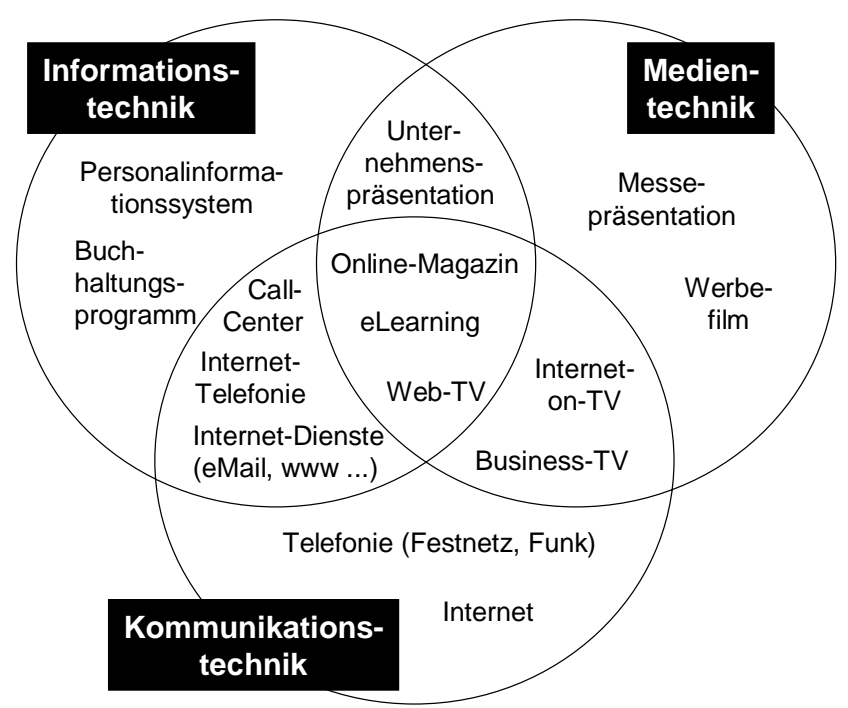

Abb. 2: Technologiekonvergenz im Bereich der IuKTechnologien [8] tsbereichen, die bisher überhaupt nicht oder kaum mit einer Branche in Beziehung standen, verändern die Wettbewerbsregeln grundlegend. Dies gilt z. B. im Sektor der Finanzdienstleistungen, wo sich in den letzten Jahren die Grenzen zwischen Versicherungen und Banken zunehmend auflösen und integrierte Finanzdienstleistungskonzerne mit einem Allfinanzangebot entstanden sind.

Doch nicht nur große Unternehmen können sich als Newcomer auf einem Markt positionieren. Kleine Firmen - ggf. im Rahmen so genannter „,virtueller Unternehmen" - sind im Zeitwettbewerb in der Lage, mit großen, etablierten Unternehmen in Konkurrenz zu treten. Innovationsfähigkeit, Entscheidungsfreudigkeit und Schnelligkeit, das Arbeiten in Netzwerken und Kooperationsfähigkeit sind die von den neuen Wettbewerbsbedingungen geforderten Qualifikationen.

\subsection{Neue Zielgruppen, neue Märkte}

Um auf ihren Märkten erfolgreich zu sein, müssen Unternehmen flexibel reagieren und sich bietende Chancen schnell nutzen. Dies erfordert seitens der Mitarbeiter die Fähigkeit zum Erkennen von Trends, Kreativität bei der Entwicklung entsprechender Produkte und Dienstleistungen und die Kompetenz, diese dem Kunden gegenüber zu kommunizieren. Basis hierfür ist die Fähigkeit, die Sicht des Kunden einzunehmen, sein Geschäft zu verstehen und seine Interessenslage antizipieren sowie mit eigenen Produkten und Leistungen unterstützen zu können.

Hierzu ist eine Kundenorientierung nicht nur im Vertrieb oder im Service, sondern über alle Wertschöpfungsstufen des Unternehmens hinweg erforderlich. Nur wer die Entwicklungstrends in seinem Abnehmermarkt detailliert kennt und sich mit seinen Kunden vernetzt, wird künftig erfolgreich sein. Kommunikation mit dem Markt und in den Markt wird zur wichtigen Aufgabe letztlich aller Unternehmensbereiche. Wer z. B. Produktideen zusammen mit seinen Kunden entwickelt, erwirbt Vorteile im Wettbewerb, die von der Konkurrenz nur langsam aufgeholt werden können.

\subsection{Anstieg des Service- und Softwareteils an der Wertschöpfung}

In zunehmenden Maße entwickelt sich Software zu einem eigenständigen Wirtschaftsgut und bildet für unternehmensinterne Abläufe und das unternehmensexterne Angebot (vor allem im Dienstleistungsbereich) 


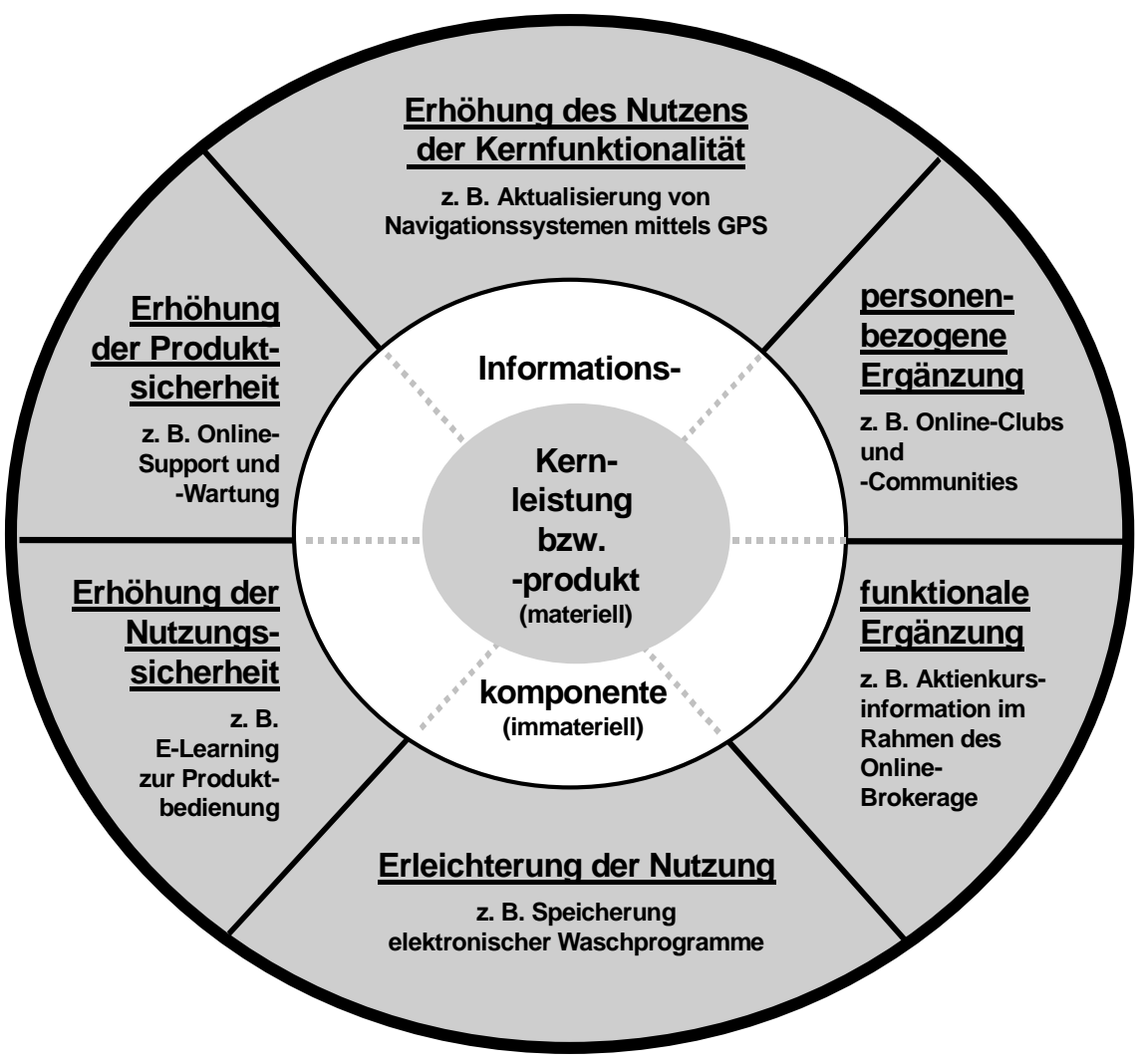

Abb. 3: Nutzen von Informationskomponenten [9].

einen neuen Produktionsfaktor. Dieser wachstumsstarke Bereich eröffnet für Unternehmen neue Chancen. Hoch integrationsfähige, flexibel anpass- und erweiterbare Hard- und Softwarekonstrukte bieten sowohl für Unternehmen der IT- Branche als auch für Anwendungsunternehmen Potenziale, um den Nutzen herkömmliche Produkte durch eine Informationskomponente anzureichern oder um eine Informationsdienstleistung zu erweitern, vgl. Abbildung 3.

Dies führt dazu, dass Informationstätigkeiten und Informationsprodukte einen immer größeren Anteil an der Wertschöpfung in den Unternehmen haben. „So besteht der Wert heutiger Personenkraftwagen zu 70\% aus „unsichtbaren“ Leistungen, wie Forschung, Entwicklung und Ausbildung. Große Teile des hierbei erarbeiteten Know-how sind in Software realisiert, aber eben nicht mehr als materielle Produktbestandteile sichtbar" $[8]$.

\subsection{Zunahme der Wissensproduktion}

Fachspezifische Kenntnisse bleiben Grundlage für ein erfolgreiches Arbeitsleben. Betrachtet man das wissenschaftliche Wissen, so sind wir heute ,mit einer Verdoppelungszeit aller wichtigen Input- und Outputgrößen (Manpower, Ressourcen, Publikationen, Pa- tente) von 10 bis 15 Jahren und mit einem Grad der Gegenwartskonzentration bis zu 90\%" [10] konfrontiert. Im außerwissenschaftlichen Bereich der Verwaltung, Massenmedien, Informationsdienste und Sicherungseinrichtungen wird die Verdoppelungszeit sogar auf nur drei bis fünf Jahre geschätzt.

Fachinformationen nehmen damit so schnell zu, dass jeder sein Wissen kontinuierlich erneuern und den rasch wechselnden Anforderungen des Arbeitsmarktes anpassen muss. Hierbei ist die Bereitschaft gefordert, Kollegen, Kunden, Marktpartner etc. am eigenen Wissen partizipieren zu lassen, genauso wie fremdes Wissen erworben und internalisiert werden muss. In den Unternehmen ist ein Wissensmanagement zur Wissensentwicklung, -verbreitung und -nutzung einzurichten, das jedoch nur dann erfolgreich sein kann, wenn es von team- und kooperationsfähigen, sozial kompetenten Mitarbeitern getragen wird (vgl. [11]) - auch dies ist eine Herausforderung an ein Lernen, welches das gesamte Berufsleben begleitet.

\subsection{Veränderte Wertesysteme}

Die heutige Arbeitswelt erfordert einen grundlegend anderen Kanon an Werten als zu Zeiten der frühen Industrialisierung, wo Fleiß, Disziplin, Gehorsam und 
Unterordnung die wesentlichen „Tugenden“ waren. In der heutigen Zeit, wo langfristig geordnete Arbeitsverhältnisse überwiegend der Vergangenheit angehören, sind allgemeine Basiskompetenzen wie Selbständigkeit und Flexibilität gefragt. Statt Unterordnung muss Eigenverantwortung übernommen werden und das Einzelkämpfertum wird durch Teamfähigkeit abgelöst (vgl. [3], S. 73f.).

Bezogen auf eine kulturelle Spannbreite von gruppenspezifischen Subkulturen bis ethnischen Landeskulturen müssen Unternehmenskulturen künftig in der Lage sein, verschiedenste Wertsysteme nicht nur zuzulassen, sondern diese auch für Effektivität und Produktivität zu nutzen. Für den Einzelnen bedeutet dies die Herausforderung, einerseits in den eigenen Werten sicher zu sein, aber andererseits auch über die Kompetenz zu verfügen, sich in wertepluralistischen Umgebungen erfolgreich bewegen zu können.

\subsection{Abnahme der Halbwertzeit des Wissens}

Die oben beschriebene Wissensexplosion geht mit einer gleichzeitigen Wissensentwertung einher. Veralterungsprozesse, insbesondere bezogen auf das Fachwissen, beschleunigen sich ständig. Je nach Fachrichtung wird von Zeiträumen von drei bis fünf Jahren gesprochen, wobei sich dies eher auf technische als auf geisteswissenschaftliche Wissensgebiete beziehen dürfte.

Die Fähigkeit, bereits erworbenes Wissen auf dem aktuellen Stand zu halten und sich darüber hinaus neues Wissen anzueignen, wird durch den Wandel der Arbeitswelt immer wichtiger. Ständige Weiterbildung wird notwendig, um dem individuellen Wissensverfall vorzubeugen.

\subsection{Verschiebung der Lerninhalte}

Die Beherrschung einer Fachmaterie stellt heute bestenfalls noch eine Eintrittskarte in die berufliche Laufbahn dar. Neben die Fachkompetenz tritt die Methodenkompetenz als überfachliche Qualifikation. Methodenkompetenz ist zu verstehen als „Fähigkeit, sich flexibel auf veränderte Arbeitsbedingungen einzustellen sowie Beherrschen von Problemlösungen und -regeln" ([12], S. 15). Beispiele für Methodenkompetenzen sind (vgl. [13], S. 105):

- Moderationstechniken,

- Projektmanagementtechniken,

- Methoden und Techniken des Qualitätsmanagements,
- Organisationsmethoden und -techniken,

- Planungsmethoden und -techniken,

- Dokumentationstechniken und

- Präsentationstechniken.

Soziale Qualifikationen und Kompetenzen (im „global village" insbesondere eine umfangreiche Sprachqualifikation) sind heute unabdingbar für den beruflichen Erfolg. Hierzu gehören (nach [13], S. 109f.)

- die Fähigkeit zur intellektuellen und emotionalen Selbstreflexion,

- Fähigkeiten des Selbstmanagement (z. B. Selbstkontrolle, Gewissenhaftigkeit oder Anpassungsfähigkeit),

- soziale Kompetenzen (z. B. Empathie und Kundenorientierung) und

- soziales Bewusstsein (z. B. Fähigkeiten zur Teamarbeit, zur Konfliktlösung, zu Kommunikation und Kooperation).

Basis für ein lebenslanges Lernen ist natürlich auch die Fähigkeit zum Lernen selbst sowie zum Aneignen neuer Denkmuster und Verhaltensweisen.

\subsection{Weiterbildung als Attraktivitätsfaktor}

Personalentwicklung wird zum unverzichtbaren Element der Zukunftssicherung eines Unternehmens und ist mittlerweile auch ganz überwiegend als solches in den Unternehmen anerkannt (vgl. [14], S. 21f.). Dies hat zur Folge, dass heute ein gesteigertes Augenmerk auf die Entwicklung und Organisation der unternehmensbezogenen Weiterbildung gelegt wird.

Unternehmen sind im Wettbewerb um die besten Arbeitskräfte zunehmend gefordert, effiziente interne Strukturen für Weiterbildung aufzubauen und ihren Mitarbeitern zur Verfügung zu stellen. Unternehmen, die ihren Bewerbern keine attraktiven Weiterbildungskonzepte anbieten können, haben zusehends Nachteile in der Personalgewinnung.

Bei der Gestaltung der Weiterbildung gilt es, unternehmensinterne und -externe Strukturelemente effektiv und effizient miteinander zu verbinden. Die Vielfalt der Weiterbildungsangebote ist gewachsen, neue Medien (z. B. E-Learning) werden als innovative Vermittlungsformen erprobt oder genutzt, immer mehr Zielgruppen werden angesprochen. Private und öffentliche Weiterbildungsorganisationen und Hochschulen finden neue Organisationsmodelle, um die Realisierung von weiterbildenden Strukturen zu gewährleisten. 


\subsection{Bevölkerungsdynamik}

Die Veränderung der Altersstrukturen in Richtung einer im Durchschnitt immer älteren Gesellschaft zeigt sich in der Entwicklung des Altersquotienten, der sich aus den 65-Jährigen und älteren Menschen in Prozent der 15- bis 64-Jährigen ergibt. Dieser Altersquotient lag in Deutschland 1999 noch bei 23,9 \%. Er wird im Jahr 2010 bei $30 \%$, im Jahr 2030 bei knapp 43 liegen (nach [6], S. 7). Dies lässt die Frage nach einer längeren Lebensarbeitszeit wieder offen werden, was offensichtlich weitere Anforderungen an ein lebenslanges Lernen stellt.

Aber nicht nur die Erwerbsarbeit ist hier anzuführen, auch der Trend in Richtung einer Arbeit ohne Erwerbshintergrund (z. B. im Rahmen eines Ehrenamtes bzw. einer Betätigung im gesellschaftlichen, kirchlichen oder sozialen Bereich) erfordert eine Qualifizierung auch nach dem Ausstieg aus dem Erwerbsleben.

\subsection{Verkürzung der Produktlebenszyklen}

Die Fähigkeit zur schnellen Produktentwicklung ist zur Grundvoraussetzung für die Wettbewerbsfähigkeit von Unternehmen auf dem Weltmarkt geworden. Dies ist das Ergebnis der sich weiter verkürzenden Produktlebenszyklen. Produkte, selbst Marken, die über zwei Generationen hinweg bekannt sind, werden im Konsumgüterbereich immer seltener. Erfolgreiche Unternehmen zeichnen sich dadurch aus, dass der Großteil ihres Umsatzes mit Produkten und Dienstleistungen erzielt wird, die nicht länger als fünf Jahre auf dem Markt sind. Hierauf müssen sich Unternehmen einstellen. An eine hocheffiziente Produktentwicklung muss sich eine reibungslose Organisation der Produktionsabläufe anschließen und in flexible Vertriebs- und Servicestrukturen münden, die wiederum der Produktentwicklung systematisch ein Feedback vom Markt geben.

Flexibilität, Kommunikation und Kooperation mit Zulieferfirmen und Kunden erfordern andere Qualifikationen als die Produktfertigung für einen anonymen Massenmarkt.

\subsection{Von der Industrie- zur Dienstleistungsgesellschaft}

Mit dem weltweiten Wandel von der Industrie- zur Dienstleistungsgesellschaft verbinden sich Hoffnungen auf Wachstumsreserven und Beschäftigungsgewinne. Allerdings ist in 19 OECD-Ländern die Beschäftigung von qualifizierten Arbeitskräften von 1970 bis 1994 zwar fast unverändert geblieben, die Beschäftigung unqualifizierter Arbeitskräfte ist jedoch stark zurück gegangen (nach [15], S. 253). Zumindest zeigt sich hierin die gewachsene Bedeutung der Qualifizierung für die Arbeitsplatzsicherheit.

Für den Wandel von der Industrie- zur Dienstleistungsgesellschaft charakteristisch ist die Verschiebung des Verhältnisses beim Einsatz von Sach- und Humankapital. In den 1920er Jahren hatte in Deutschland das Sachkapital (gemessen am Bruttoanlagevermögen) noch einen fünfmal höheren Wert als das Humankapital (gemessen an den Ausbildungskosten). Bis 1989 hat sich diese Relation auf 2,2:1 angenähert (nach [15], S. 252f.).

Der Umgang mit und die Nutzung von Information wird in der Dienstleistungsgesellschaft eine wesentliche Schlüsselqualifikation darstellen. Informationsverhalten und Informationskultur werden in den Unternehmen wichtige Themen und stellen ebenso wie die anderen Faktoren neue Anforderungen an die Motivation, die Prozesse und die Inhalte im Rahmen eines lebenslangen Lernens.

Auch wenn sich - wie beispielhaft gezeigt - für die Faktoren jeweils spezielle Indikatoren finden lassen und damit der Eindruck einer trennscharfen Abgrenzung entstehen kann, so bestehen doch durchaus enge Zusammenhänge zwischen den einzelnen Bestimmungsfaktoren des lebenslangen Lernens. Beispielsweise ist die Informatisierung als Durchdringung aller Lebens- und Handlungsbereiche mit Informations- und Kommunikationstechnologien wesentliches Merkmal der Informationsgesellschaft. Sie beruht jedoch auf weltumspannenden Informationsnetzwerken, die wiederum Ausdruck der Globalisierung sind (vgl. [16], S. 153). Die Verkürzung von Produktlebenszyklen fordert die Entwicklung innovativer Produkte und Leistungen. Oder die Abnahme der Halbwertzeit des Wissens führt in den Unternehmen zum Aufbau von Strukturen und Verfahren der Weiterbildung und des Wissensmanagements.

\subsection{Fazit}

Durch die beschriebenen Entwicklungen steigt nicht nur der Ausbildungs-, sondern vor allem der Weiterbildungsbedarf. Weiterbildung ist im globalen, internationalen, nationalen und regionalen Wettbewerb als jeweils gesellschaftliche Aufgabe der betreffenden Un- 
ternehmen, öffentlichen und privaten Weiterbildungsträger und verstärkt auch Hochschulen anzusehen. Sie gibt dem Einzelnen die Möglichkeit, sein Humankapital vor der Entwertung zu bewahren und seine neu erworbenen Kenntnisse der Gesellschaft zur Verfügung zu stellen.

Für Unternehmen stellt sich die Aufgabe, die individuellen Weiterbildungsbedarfe der Mitarbeiter und des Unternehmens $\mathrm{zu}$ integrieren und im Rahmen eines systematischen Konzepts der Personalentwicklung zu decken. Hierbei darf keine Begrenzung - etwa auf so genannte „High- Potentials“ - stattfinden, sondern lebenslanges Lernen ist Bedürfnis und Anspruch aller Mitarbeiter in allen Unternehmensbereichen und über alle hierarchischen Ebenen hinweg (vgl. [17], S.24).

\section{Zusammenfassung}

Lebenslanges Lernen ist eine der wichtigsten Herausforderungen, denen sich fortgeschrittene, moderne Gesellschaften gegenüber sehen. Ein lebenslanges Lernen muss strukturiert und inhaltlich gestaltet werden. Voraussetzung hierfür ist die Kenntnis der Bestimmungsfaktoren, aus denen die Erfordernis eines lebenslangen Lernens resultiert. Diese Bestimmungsfaktoren sind:

- Europäisierung und Globalisierung der Unternehmensgeschäfte,

- Entwicklung der Informationsgesellschaft,

- Internationalisierung des Wettbewerbs,

- Auftauchen neuer Zielgruppen und neuer Märkte,

- Eintritt neuer Wettbewerber in die Märkte,

- Anstieg des Service- und Softwareteils an der Wertschöpfung,

- veränderte Wertesysteme,

- Zunahme der Wissensproduktion,

- Abnahme der Halbwertzeit des Wissens,

- Verschiebung der Lerninhalte,

- Weiterbildung als Attraktivitätsfaktor bei der Personalgewinnung,

- Auswirkungen der Bevölkerungsdynamik,

- Verkürzung der Produktlebenszyklen und

- Entwicklung von der Industrie- zur Dienstleistungsgesellschaft.

Aus diesen Bestimmungsfaktoren ergeben sich jeweils spezifische Anforderungen an Weiterbildungsmaßnahmen bzw. an die Personalentwicklung eines Unternehmens insgesamt. Eine kontinuierliche Weiterbildung betrifft alle Mitarbeiter in allen Unternehmensbereichen, über alle hierarchischen Ebenen hinweg.

\section{Literatur}

1. Henning, Klaus; Staufenbiel, Joerg E.: Berufsplanung für Ingenieure. Köln: Staufenbiel 1999.

2. Europäische Kommission, Generaldirektion Bildung und Kultur: Lebenslanges Lernen - bald Wirklichkeit für alle. Luxemburg: Amt für Veröffentlichungen der Europäischen Gemeinschaften 2002.

3. Klotz, Michael; Förtsch, Frank; Hugler, Silke u. a.: Herausforderungen aus Umweltdynamik und Arbeitsweltwandel. In: Zdrowomyslaw, Norbert; Rethmeier, Bernd (Hrsg.): Studium und Karriere - Karriere- und Berufsplanung, Erfolg und Worklife-Balance. München-Wien: Oldenburg 2001, S. 63-99.

4. Siebert, Horst: Außenwirtschaft. 7. Aufl., Stuttgart: Luciuc und Lucius 2000.

5. Went, Robert: Ein Gespenst geht um ... Globalisierung: eine Antwort. Zürich: Orell Füssli 1997.

6. Institut der Deutschen Wirtschaft Köln: Deutschland in Zahlen 2002. Köln: Dt. Instituts-Verlag 2002.

7. Dresdner Bank Wirtschaft International, Dezember 2002 [www.dresdner-bank.de/meta/kontakt/ 01_economic_research/].

8. Klotz, Michael: Informationsgesellschaft. In: Schildhauer, Thomas (Hrsg.): Lexikon Electronic Business. München-Wien: Oldenburg 2003, S. 157-161.

9. Klotz, Michael: Informationsgut. In: Schildhauer, Thomas (Hrsg.): Lexikon Electronic Business. MünchenWien: Oldenburg 2003, S. 161-165.

10. Spinner, Helmut F.: Die Wissensordnung - Ein Leitkonzept für die dritte Grundordnung des Informationszeitalters. Opladen: Leske + Budrich 1994.

11. Klotz, Michael; Strauch, Petra: Wissensmanagementsysteme: Komponenten und Erfolgsfaktoren für den Einsatz. In: Informationsmanagement - Herausforderungen und Perspektiven, 3. Liechtensteinisches Wirtschaftsinformatik-Symposium an der FH Liechtenstein, hrsg. von Bernd Bitzelmaier, Stephan Geberl und Siegfried Weinmann, Stuttgart-Leipzig-Wiesbaden: Teubner 2001, S. 79-94.

12. Heberer, Juliane; Grap, Rolf: Betriebliche Weiterbildung in kleinen und mittelständischen Unternehmen - Methoden und Vorgehensweise. Herzogenrath: Verlag der GOM 1995.

13. Mertens, Ralf; Grode, Andrea; Hönger, Vivian u. a.: Anforderungen an Mitarbeiter und Personalmanagement. In: Zdrowomyslaw, Norbert; Rethmeier, Bernd (Hrsg.): Studium und Karriere - Karriere- und Berufsplanung, Erfolg und Worklife-Balance. München-Wien: Oldenburg 2001, S. 101-147.

14. Mühlemeyer, Peter; Flenner, Vanessa: Umsetzung des „Managementinstruments Personalentwicklung“ in der betrieblichen Praxis - Ergebnisse einer explorativen Studie an der Fachhochschule Worms. In: Personal 2002, Heft 7, S. 20-24. 